\title{
Moving Object and Shadow Detection Algorithm Using Lab Color Space
}

\author{
Ju Zhiyong ${ }^{1, a}$, He Xiaolei ${ }^{1, b}$, Wang Chaonan ${ }^{1, c}$
}

1 School of Optical-Electrical and Computer enginnering, University of Shanghai for Science and Technology, Shanghai, China

ajuzy@usst.edu.cn b15850695057@163.com 'chaonanw1012@163.com

Keywords: Lab space; background subtraction; Color algorithm; moving object detection; shadow detection

\begin{abstract}
Taking advantage of independence of chromaticity and brightness in Lab space, this paper transplants the idea separating the brightness distortion from the chromaticity distortion mentioned in the Color Algorithm into Lab space, redefine the brightness distortion and chromaticity distortion. Based on the new computational color model, the paper simplifies and improves the formulas relevant. Besides, aiming at the problem of outdoor illumination change, this paper also updates the brightness of outdoor background combined with frame difference in time. The experiment proved that the improved algorithm shows good effect of detecting moving target and shadow. Compared with the original algorithm, the improved algorithm can better adapt to detection of vehicles and shadow outdoor.
\end{abstract}

\section{Introduction}

The capability of extracting moving objects from a video sequence is a fundamental and crucial function of a visual system. This useful function applies to many systems including traffic monitoring, human body detection or video surveillance[1]. Background subtraction is the most typical one among various detection algorithms, which can broadly be divided into 3 categories: pixel-based, region-based and frame-based[2]. The main idea of pixel-based algorithms is aimed at forming a statistical background model for each pixel separately, among which the most popular technique is Gaussian Mixture Model(GMM) as well as Kernel densities. In the paper[3], each pixel was assumed normally distributed and then $\mathrm{KLD}$ (Kullback-Leibler divergence) could be computed between a background pixel and a current pixel with the help of Gaussian mixture model , which is useful enough to classify each pixel. In the paper[4], the algorithm uses multiple background models of expected illumination changes followed by both pixel and frame on both RGB and YCbCr color spaces. Concerning region-based techniques, in the paper[5], its work exploits local spatial relationships among pixels. On the contrary, frame-based methods normally create statistical background models for the entire frame, Many of which are based on a shading model. It need to calculate the ratio of intensities between an input image and the reference frame or background model[2]. In the paper[6], the distribution of the shadow is modeled with a GMM and then develop the Statistical Illumination model. In the paper[7],the authors propose a unified model sharing framework for moving object detection. They exploit the spatial-temporal correlation across different pixels and establish a many-to-one correspondence by model sharing between pixels. Each pixel is labeled into foreground or background by searching an optimal matched model in the neighborhood. In the paper[8], the authors propose a uniform model named auto regression and texture (ART, Auto regression and texture), which is composed of a real-time background modeling framework detecting moving objects precisely and a sensitive texture background model that can exactly track outdoor illumination variation in real time.

The algorithm proposed in the paper[9] is one of the pixel-based categories and is based on a proposed computational color model separating the brightness from the chromaticity component in the three-dimensional RGB color space. The authors decompose the distortion into two components, which is brightness distortion and chromaticity distortion. This two components is the main basis of pixel classification. Actually it is dependent between brightness and color in the RGB space. It 
means that the decomposition of color and brightness is measured artificially by complex formulas. In this paper, we take the advantage of the characteristic that the brightness and color are independent of each other in the Lab color space and propose a novel algorithm related to the original Color Algorithm. In accordance with the comparison between the improved and the original, the improved algorithm shows the better adaptability of scene. It also can accurately detect and mark shadows.

\section{Computational Color Model of the Original Algorithm}

The paper[9] proposed a computational color model in the three-dimensional RGB color space, in which the background image is pixel-wise modeled and statistical. The model defies that $E_{i}$ represents an expected color of a given $i^{\text {th }}$ pixel and $I_{i}$ represents the color value of the pixel in a current image[9].

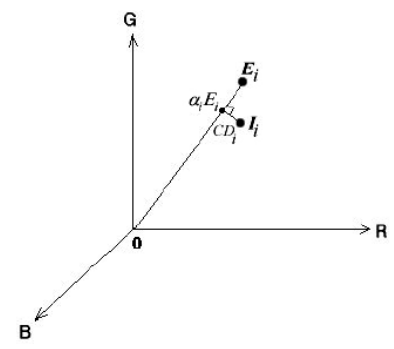

Fig.1 Computational color model of the original algorithm

Consider a pixel $I$ in the image; let $E_{i}=\left[E_{R}(i), E_{G}(i), E_{B}(i)\right]$ represent the pixel's expected RGB color in the reference or background image. The line $\mathrm{OE}_{\mathrm{i}}$ passing through the origin and the point $E_{i}$ is called expected chromaticity line. Next, let $I_{i}=\left[I_{R}(i), I_{G}(i), I_{B}(i)\right]$ denote the pixel's RGB color value in a current image that we want to subtract from the background. Basically, we want to measure the distortion of Ii from Ei. The difference between $\mathrm{I}_{\mathrm{i}}$ and $\mathrm{E}_{\mathrm{i}}$ is decomposed into brightness $\left(\alpha_{\mathrm{i}}\right)$ and chromaticity $\left(\mathrm{CD}_{\mathrm{i}}\right)$ components[9].

The brightness distortion is a scalar value that brings the observed color close to the expected chromaticity line. It is obtained by minimizing

$$
\emptyset\left(\alpha_{i}\right)=\left(I_{i}-\alpha_{i} E_{i}\right)^{2}
$$

$\alpha_{i}$ represents the pixel's strength of brightness with respect to the expected value. $\alpha_{i}$ is 1 if the brightness of the given pixel in the current image is the same as in the reference image. $\alpha_{i}$ is less than 1 if it is darker, and greater than 1 if it becomes brighter than the expected brightness.

Chromaticity distortion is defined as the orthogonal distance between the observed color and the expected chromaticity line. The color distortion of a pixel $i$ is given by

$$
\mathrm{CD}_{\mathrm{i}}=\left\|\mathrm{I}_{\mathrm{i}}-\alpha_{\mathrm{i}} \mathrm{E}_{\mathrm{i}}\right\|
$$

\section{Improvement of Color Algorithm}

\subsection{Computational Color Model in the Lab Space}

In this paper, we propose a new computational model in the Lab color space. Consider two pixels $B_{i}$ and $C_{i}$.Let $B_{i}=\left[L_{b}(i), a_{b}(i), b_{b}(i)\right]$ represent a pixel in a background image statically pixel-wise modeled. $L_{b}(i) 、 a_{b}(i) 、 b_{b}(i)$ separately denote three channel value of Lab space. Similarly, let $\mathrm{C}_{\mathrm{i}}=\left[\mathrm{L}_{\mathrm{c}}(\mathrm{i}), \mathrm{a}_{\mathrm{c}}(\mathrm{i}), \mathrm{b}_{\mathrm{c}}(\mathrm{i})\right]$ represent a pixel in a current image.

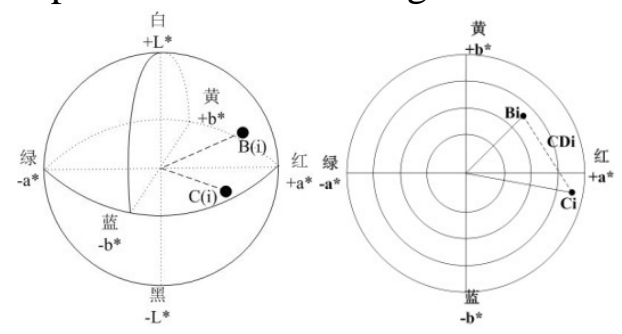

Fig.2 Computational color model in the Lab space

In this model, the chromaticity distortion is defined as the Euclidean distance between $\mathrm{B}_{\mathrm{i}}$ and $\mathrm{C}_{\mathrm{i}}$ 
in the $\mathrm{a}^{*} \mathrm{~b}^{*}$ color plane projection. The formula of chromaticity distortion is given by

$$
\mathrm{CD}_{\mathrm{i}}=\sqrt{\left(\mathrm{a}_{\mathrm{b}}(\mathrm{i})-\mathrm{a}_{\mathrm{c}}(\mathrm{i})\right)^{2}+\left(\mathrm{b}_{\mathrm{b}}(\mathrm{i})-\mathrm{b}_{\mathrm{c}}(\mathrm{i})\right)^{2}}
$$

Considering that $\mathrm{L}$ channel of Lab space is on behalf of brightness, we difine that brightness distortion is the difference between $B_{i}$ and $C_{i}$ on the $L$ channel. The fomula is given like this below

$$
\mathrm{BD}_{\mathrm{i}}=\mathrm{L}_{\mathrm{b}}(\mathrm{i})-\mathrm{L}_{\mathrm{c}}(\mathrm{i})
$$

The brightness of the given pixel in the current image is the same as in the reference image if $\mathrm{BD}_{\mathrm{i}}$ is zero. It is darker if $\mathrm{BD}_{\mathrm{i}}$ is negative, and it is brighter than the pixel in current image if $\mathrm{BD}_{\mathrm{i}}$ is positive. It is the basis of classifying shadow pixels.

We use the first frame of the image sequences (not including the moving target) as the initial background model and update the brightness of background in real time to adapt illumination changes outdoor.

\subsection{Adjustment of Paraments}

In order to ensure the threshold of $\mathrm{BD}$ and $\mathrm{CD}$ be in the range of each channel of the space and the details of image texture is not lost as far as possible, we normalize the paraments BD and CD. We set two normalization factors, a and b.

a represents the RMS of brightness distortion of the whole current image, which is given by

$$
\mathrm{a}=\operatorname{RMS}\left(\mathrm{BD}_{\mathrm{i}}\right)=\sqrt{\frac{\sum_{\mathrm{i}=0}^{\mathrm{N}} \mathrm{BD}_{\mathrm{i}}^{2}}{\mathrm{~N}}}
$$

Similarly, b represents the RMS of chromaticity distortion of the whole current image, which is given by

$$
\mathrm{b}=\operatorname{RMS}\left(\mathrm{CD}_{\mathrm{i}}\right)=\sqrt{\frac{\sum_{\mathrm{i}=0}^{\mathrm{N}} \mathrm{CD}_{\mathrm{i}}^{2}}{\mathrm{~N}}}
$$

$\mathrm{N}$ represents the number of pixels in the image, and $\mathrm{i}=1,2,3 \ldots, \mathrm{N}$.

Then we rescale the $\mathrm{BD}_{\mathrm{i}}$ and $\mathrm{CD}_{\mathrm{i}}$, let

$$
\begin{aligned}
& \widehat{B D}_{1}=\frac{\mathrm{BD}_{\mathrm{i}}}{\mathrm{a}} \\
& \widehat{\mathrm{CD}}_{1}=\frac{\mathrm{CD}_{\mathrm{i}}}{\mathrm{b}}
\end{aligned}
$$

be the normalized brightness distortion and normalized chromaticity distortion respectively.

\subsection{Pixel Classification and Threshold Selection}

In this paper, a pixel is classified into one of three categories background pixel、shadow pixel、 moving object pixel, which is given by the following decision procedure.

(1) If it has similar chromaticity to that of the same pixel in the background, the pixel should be classified into background pixel.

(2) If it has lower brightness than that of the same pixel in the background image, the pixel should be classified into shadow pixel.

(3) If the pixel has chromaticity different from that of in the background image, it should be classified into the moving object pixel.

The procedure is concluded as

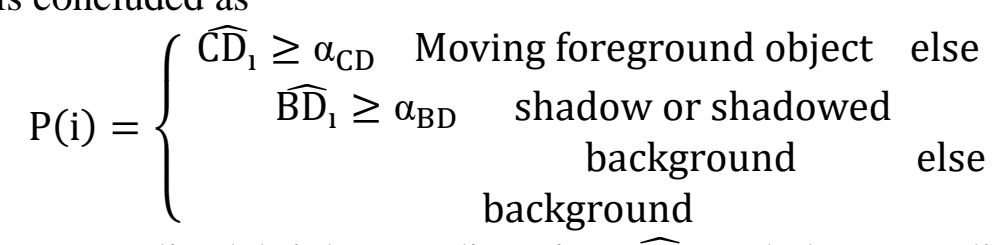

The integrams of the normalized brightness distortion $\widehat{\mathrm{BD}}_{1}$ and the normalized chromaticity distortion $\widehat{C D}_{1}$ shows that both of the two distribution are not any of existing distributions. Thus we take experients both outdoor and indoor and analyse statistically. According our experiments and analysis of hundreds images, two appropriate thresholds $\alpha_{C D}$ and $\alpha_{B D}$ are selected artificially for the circumstances outdoor and indoor respectively. The threshold of artificial selection may not be the most accurate, but the effect of classification is very obvious and reduce the possibility of noise pixel mistaken into other kind of categories. 


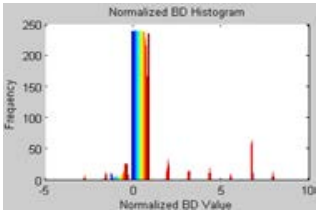

(a)

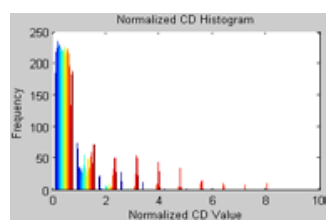

(b)

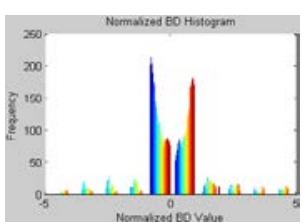

(c)

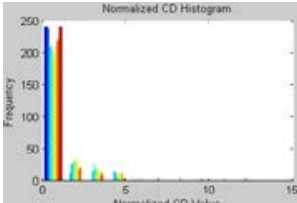

(d)

Fig.3 Integrams of $\widehat{\mathrm{BD}}_{1}$ and $\widehat{\mathrm{CD}}_{1}$ outdoor(a and b of Fig.5 1(a)) and indoor(c and d of Fig.6 $1(\mathrm{a}))$

\subsection{Strategy for Background Brightness Updating}

The reasons for illumination changes outdoor include: slow light change over time; rapid illumination changes caused by weather changes, such as rapid cloud occlusion. Accurate target detection results require that the background model has good adaptability to the outdoor illumination changes[8]. Aiming at the illumination change outdoor, we adjust the brightness of the background model in the $\mathrm{L}$ channel of Lab space in time. The formula is shown as follows.

$$
\begin{gathered}
\widehat{\mathrm{L}_{k}}=\mathrm{L}_{1}+\sum_{\mathrm{i}=2}^{\mathrm{k}} \alpha_{\mathrm{j}} \mathrm{M}_{\mathrm{i}} \\
\mathrm{M}_{\mathrm{j}}=\mathrm{L}_{\mathrm{j}}-\mathrm{L}_{\mathrm{j}-1}
\end{gathered}
$$

$L_{1}$ is the $L$ channel data of the initial background frame; $M_{i}$ is the $L$ channel difference of two adjacent frame; $\alpha_{i}$ is a background updating parameter; $\widehat{\mathrm{L}_{\mathrm{k}}}$ is the $\mathrm{L}$ channel data of the $\mathrm{k}^{\text {th }}$ frame after the background updating.

About determinating the background updating parameter, firstly we should distinguish the type of a pixel related. It means that the current pixel is the target pixel or non-target pixel (non-target pixels including background pixels and shadow pixels). If the current pixel is a non-target pixel, we update the L channel data of the pixel; otherwise we do not. Because we only update the brightness of the background model and it is independent between brightness and chromaticity in Lab color space, we can exploit $\widehat{C D}_{1}$ to distinguish the type of pixels.

The formula for determining the background update parameter is given by

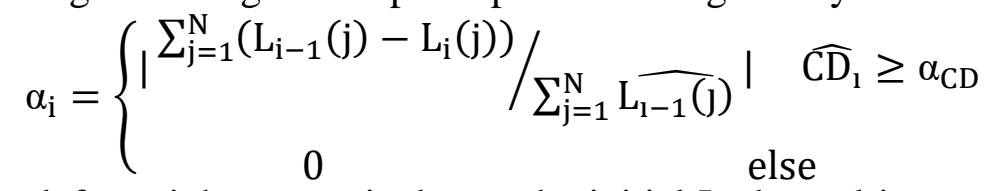

Images in Figure 4 from left to right respectively are the initial L channel image of background frame、L channel image after updated $\widehat{\mathrm{BD}}_{1}$ image after background subtraction using the initial background image $\widehat{\mathrm{BD}}_{1}$ image after background subtraction using the new background image. Comparison shows that after updating the brightness the contour of vehicle body is more clear and illumination updating does not affect the shadow detection.
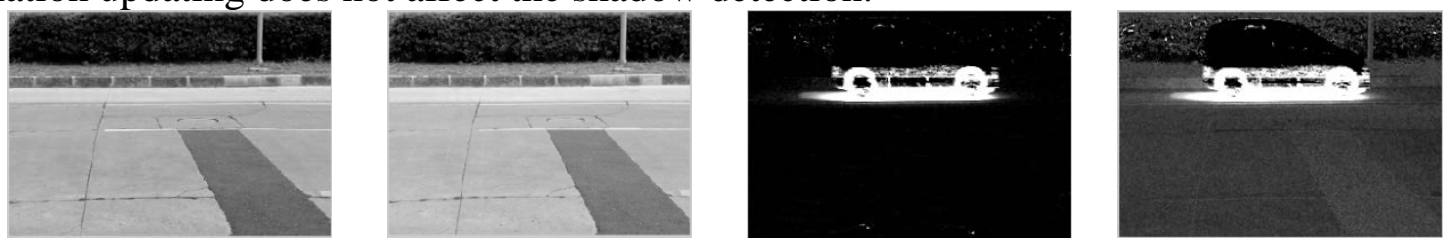

Fig.4 L channel images and $\widehat{\mathrm{BD}}_{1}$ images before and after background updating

For outdoor vehicle video sequences, take the initial background frame (1(a) in Fig.5), the 222 frame (1(b) in Fig.5) and the 870 frames (2(b) Fig.5) as an example. Because this method is aimed at the global illumination change, we cut a region of $32 * 24$ in respect of the whole image. Let $\beta$ org represents the average brightness of the initial background region, $\beta$ cur represents the average brightness of background region in the current image and $\beta$ up represents the average brightness of the background region after updating. The table 1 followed shows the error percentage between $\beta$ org and $\beta$ up and the good adaptability of our brightness update method.

$$
\text { Error Percentage }=\frac{\beta_{\mathrm{up}}-\beta_{\mathrm{org}}}{\beta_{\mathrm{cur}}} \times 100 \%
$$


Table 1 Error analysis of background brightness updating

\begin{tabular}{|c|c|c|}
\hline & 222th Frame & 870th Frame \\
\hline$\beta_{\text {org }}$ & 168.75 & 168.75 \\
\hline$\beta_{\text {cur }}$ & 168.73 & 170.48 \\
\hline$\beta_{\text {up }}$ & 169.01 & 170.96 \\
\hline Error Percentage & $0.17 \%$ & $0.28 \%$ \\
\hline
\end{tabular}

\section{Experimental Results and Analysis}

In this paper, the size of video sequence frames is $320 * 240$, and the processing environment is 32-bit win7 system 、frequency $1.8 \mathrm{GHz} 、 4 \mathrm{G}$ memory and Matlab2014a. Algorithms are tested on 870 frames outdoor road scene and 350 frames indoor scene. Fig.5、 Fig6 and Fig.7 show three groups of experimental results. Fig. 5 and Fig. 6 are the comparison between our method and the original Color algorithm. The six colum images in Fig.5 and Fig.6 are respectively initial background frame, current frame, pixel classification results of our method, the detection effect of the original color algorithm, the object detected with the original and the object detected with our method. Fig.7 is the comparison between our algorithm and other three traditional pixel-based algorithms. We mark the moving object pixels in blue, the shadow pixels in red and the background pixels in black. To the contrary, in the original Color algorithm, the moving object pixels are marked in red and the shadow pixels are marked in blue and the background pixels are marked in black.

These two groups in Figure 5 is a road scene outdoor. Experimental results show that our algorithm can accurately detect different color and model vehicles, and the shadows can be identified effectively. Although the original algorithm can recognize the contour of vehicle objects, the shadow is labeled as the moving part because of the dependence between brightness and chromaticity in RGB space. It indicates that it is mutual dependent between brightness and chromaticity of the original color algorithm. Brightness distortion and chromaticity distortion defined in our algorithm is truly independent and its adaptability to vehicles and shadows outdoor is better. However another truth is that the original preserves details better than ours. $\left(\alpha_{\mathrm{CD}}=0.70\right.$, $\left.\alpha_{\mathrm{BD}}=0.65\right)$

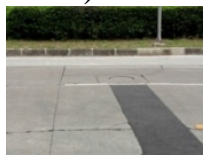

1(a)

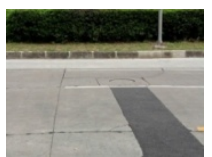

2(a)

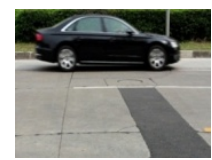

1(b)

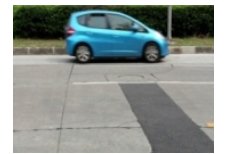

2(b)

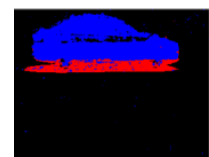

1(c)

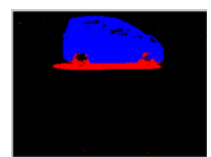

2(c)

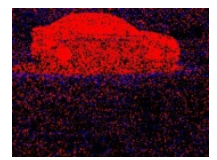

1(d)

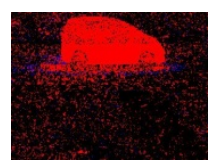

2(d)

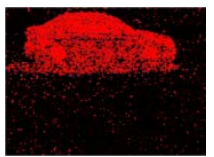

1(e)

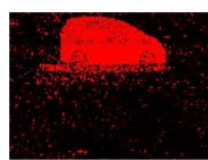

2(e)

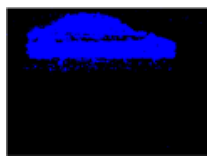

1(f)

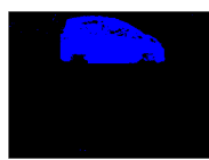

2(f)

Fig.5 Comparison of two groups of images outdoor

There are two groups of images indoor in Figure 6, and the difference between them is that the forth group is a scene with much tiles on the floor which can cause mirror reflection. The experiments show that there is no law of the position of the specular reflection. Therefore some unstable regions in the background image are incorrectly labeled as the moving object parts. So our algorithm needs to be improved in a mirror or similar scene. However, compared with the original algorithm, our method can label the upper human body more completely without morphological operations. $\left(\alpha_{\mathrm{CD}}=1.20, \alpha_{\mathrm{BD}}=0.15\right)$ 


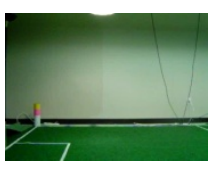

1(a)

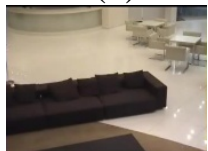

2(a)

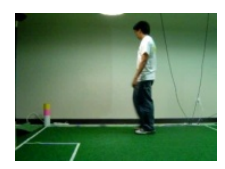

1(b)

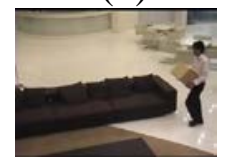

2(b)

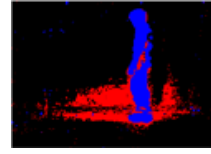

1(c)

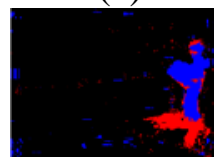

2(c)

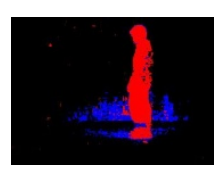

1(d)

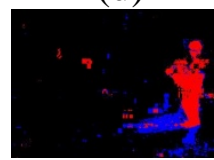

2(d)

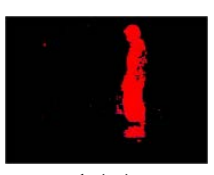

1(e)

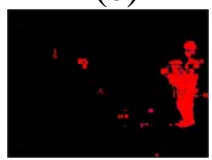

2(e)

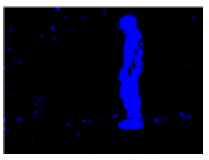

1(f)

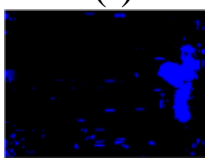

2(f)

Fig.6 Comparison of two groups of images indoor

In the last group, two vehicle images outdoor are used to compare the proposed algorithm and other three traditional pixel-based algorithms. GMM3 method classifies the dark part in the police car door into shadow and other two kind of frame difference methods can't detect objects completely. The comparison shows that vehicles and shadows are both more complete labeled using our algorithm than other three methods. The detection effect of our algorithm is better.

GMM3

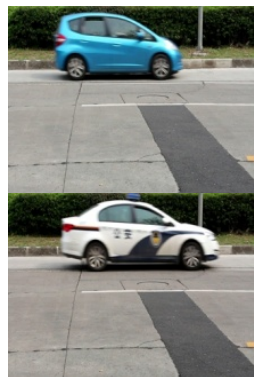

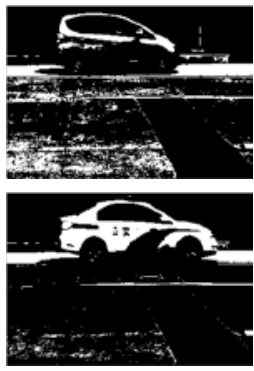

$$
\text { 2-frame }
$$

difference

3 -frame
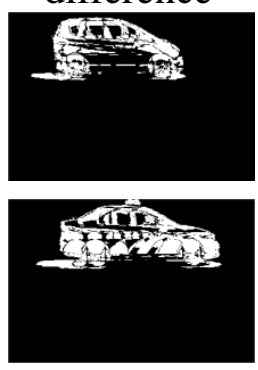

Our method

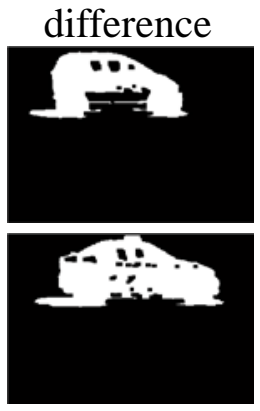

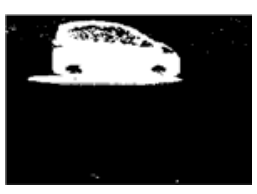

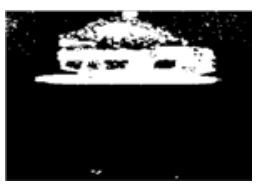

Fig.7 Comparison between our method and three traditional algorithm

\section{Conclusion}

In this paper, we use the idea of color algorithm and the independence between brightness and chromaticity in Lab color space to redefine formulas of chromaticity distortion and brightness distortion. Experiments prove that our method is more adaptive to the vehicle and shadow detection outdoor and can label the upper human body more completely without morphological operations. Moreover compared with the original one, we add the background brightness updating method so that it is more adaptive to the global outdoor illumination changes. However our algorithm still needs to be improved in a mirror or similar scene and details preservation.

\section{Reference}

[1]. Hasan Sajid , Sen-Ching Samson Cheung. Background Subtraction Under Sudden Illumination Change. 2014 IEEE 16th International Workshop on Multimedia Signal Processing (MMSP), Sep. 22-24, 2014, Jakarta, Indonesia. 978-1-4799-5896-2/14/\$31.00@2014 IEEE.

[2]. L. P. J. Vosters, C. Shan, and T. Gritti. Background subtraction under sudden illumination changes. In Proceedings of the 2010 7th IEEE International Conference on Advanced Video and Signal Based Surveillance, AVSS '10, pages 384-391,2010.3

[3]. Dileep Kumar Yadav , Karan Singh.A combined approach of Kullback-Leibler divergence and background subtraction for moving object detection in thermal video. Infrared Physics \& Technology 76 (2016) 21-31.

[4]. Hasan Sajid, Sen-Ching Samson Cheung. Background Subtraction Under Sudden Illumination Change. 2014 IEEE 16th International Workshop on Multimedia Signal Processing (MMSP), Sep. 22-24, 2014, Jakarta, Indonesia. 978-1-4799-5896-2/14/\$31.00 @2014 IEEE. 
[5]. Y. Sheikh and M. Shah. "Bayesian modeling of dynamic scenes for object detection". IEEE Transactions on Pattern Analysis and Machine Intelligence, 27(11):1778-1792, 2005.

[6]. J. Pilet, C. Strecha, and P. Fua."Making background subtraction robust to sudden illumination changes". In ECCV (4), pages 567-580, 2008.

[7]. Yingying Chen, Jinqiao Wang, MinXu, Xiangjian He, Hanqing Lu. A unified model sharing framework for moving object detection. Signal Processing 124(2016)72-80.

[8]. ZHAO Xu-Dong, LIU Peng, TANG Xiang-Long, LIU Jia-Feng1. Background Modeling Adaptive to Outdoor Illumination Variation and Foreground Detection Approach[J]. ACTA AUTOMATICA SINICA.2011-8(37):915-922.

[9]. Thanarat Horprasert, David Harwood, Larry S. Davis. A Statistical Approach for Real-time Robust Background Subtraction and Shadow Detection. Proc Int Conf Computer Vision. IEEE Frame Rate Workshop, 1999:1—19. 